\title{
BMP9-Induced Osteogenetic Differentiation and Bone Formation of Muscle-Derived Stem Cells
}

\author{
Li Xiang, Chen Liang, Ke Zhen-Yong, Yin Liang-Jun, and Deng Zhong-Liang \\ Department of Orthopaedic Surgery, The Second Affiliated Hospital, Chongqing Medical University, No. 76 Linjiang Road, Yuzhong \\ District, Chongqing 400010, China \\ Correspondence should be addressed to Deng Zhong-Liang, deng7586@gmail.com
}

Received 15 July 2011; Accepted 26 October 2011

Academic Editor: Ken-ichi Isobe

Copyright ( 2012 Li Xiang et al. This is an open access article distributed under the Creative Commons Attribution License, which permits unrestricted use, distribution, and reproduction in any medium, provided the original work is properly cited.

\begin{abstract}
Efficient osteogenetic differentiation and bone formation from muscle-derived stem cells (MDSCs) should have potential clinical applications in treating nonunion fracture healing or bone defects. Here, we investigate osteogenetic differentiation ability of MDSCs induced by bone morphogenetic protein 9 (BMP9) in vitro and bone formation ability in rabbit radius defects repairing model. Rabbit's MDSCs were extracted by type I collagenase and trypsin methods, and BMP9 was introduced into MDSCs by infection with recombinant adenovirus. Effects of BMP9-induced osteogenetic differentiation of MDSCs were identified with alkaline phosphatase (ALP) activity and expression of later marker. In stem-cell implantation assay, MDSCs have also shown valuable potential bone formation ability induced by BMP9 in rabbit radius defects repairing test. Taken together, our findings suggest that MDSCs are potentiated osteogenetic stem cells which can be induced by BMP9 to treat large segmental bone defects, nonunion fracture, and/or osteoporotic fracture.
\end{abstract}

\section{Introduction}

Skeletal muscles are always regarded as the source of satellite stem cells or muscle precursor cells. These kinds of stem cells are at the quiescent condition under normal circumstances and activated when the repairing of muscle tissue is needed. They will be differentiated and blended together into new muscle fibers, reaching the purpose of repairing defected muscles as a result [1]. In recent years, studies have shown that there is also another kind of stem cells in skeletal muscle, called muscle-derived stem cells (MDSCs). They have mesenchymal stem cells-like differentiation potential [2], and the ability of being differentiated into several types of terminal cells is maintained. Instead of being differentiated into muscle cells, MDSCs can also be differentiated into other type of cells, such as hematopoietic cells [3], osteoblasts [4], and chondroblasts [5] under particular conditions. Therefore, the potential of MDSCs as candidate seed cells in osteogenetic tissue engineering has been paid much more attention than before.

Bone morphogenetic proteins (BMPs) belong to one of the $\beta$-superfamily members of transforming growth factors
(TGFs); BMPs are a kind of multifunctional growth factors and are one of the most potent osteogenetic growth factors [6-8]. BMP9 belongs to BMPs family and is expressed in liver [9]. BMP9 possible roles include inducing and maintaining the cholinergic phenotype of embryonic basal forebrain cholinergic neurons, inhibiting hepatic glucose production and inducing the expression of key enzymes of lipid metabolism, and stimulating murine hepcidin 1 expression [10-12]. Although the functional role of BMP9 in the skeletal system remains to be fully understood, the potent osteogenic activity of BMP9 suggests that it may be used as one of the most effective bone regeneration agents compared with other BMPs, such as BMP2, BMP4, or BMP7 [13, 14].

Presently, among the overwhelming majority of many osteogenetic studies of BMPs, mouse mesenchymal stem cells (such as $\mathrm{C} 3 \mathrm{H} 10$ ), mouse embryonic fibroblasts (MEFs), and bone marrow stromal cells (BMSCs) are subjected as studying objects. However, there have been no literature reports on whether BPM9 can induce osteogenetic differentiation of muscle-derived stem cells. Therefore, in this paper, we have demonstrated the potential osteogenetic ability of MDSCs induced by BMP9 in vitro and in vivo testing. 


\section{Materials and Methods}

2.1. Materials. BMP9, BMP2, and GFP expression adenoviruses (AdBMP9, AdBMP2, and Ad-GFP) were provided by Dr. He (Molecular Oncology Laboratory, Medical Center, the University of Chicago, USA) and amplified in our laboratory. Hank's solution, DMEM culture medium, and high-quality fetal calf serum (FBS) were used in cells culture (Hyclone Company). Alkaline phosphatase (ALP) staining kit and quantitative testing kit were purchased from BD Company. Alizarin red $\mathrm{S}$ staining kit, type I collagenase, trypsin, polylysine, and vitamin C were purchased from Sigma Company. Anti-Sca-1 antibody (Wuhan Boster Biological Technology Co., Ltd.) and nanohydroxyapatite/polyamide bone cement were provided by Research Center of Nano-biomaterials, Sichuan University.

Healthy rabbits with an average age of 6-8 weeks, as the experimental animal, were provided by the Experimental Animal Center, Chongqing Medical University.

\subsection{Methods}

2.2.1. Separation and Cultivation of MDSCs. After anesthesia, muscle strips $(2 \mathrm{~cm} \times 0.8 \mathrm{~cm} \times 0.5 \mathrm{~cm}$, approximately $5 \mathrm{~g})$ were cut from the rabbits and then placed into a sterilized bottle. Carry out sequential digestion by two-step method (type I collagenase and trypsin methods), then filter through no. 100, 200, and 400 stainless steel screens, and aspirate the obtained cells into a $100 \mathrm{~mL}$ culture bottle (PP1). $9 \mathrm{~mL}$ of DMEM culture solution (contained $100 \mathrm{~mL} / \mathrm{L}$ fetal calf serum) was added. $1 \mathrm{~h}$ later, transfer the cell suspension into another culture dish (PP2) by the differential-rate walladhering growth method. Hereafter, repeat the procedure above to obtain PP3, PP4, PP5, and PP6 every $24 \mathrm{~h}$.

2.2.2. Identification of MDSCs. MDSCs were fixed with acetone and prepared for immunohistochemical staining analysis at 48 hours after subculture. Immunohistochemical staining was subjected with mouse anti-rabbit Sca-1 monoclonal antibodies.

2.2.3. ALP Staining and ALP Activity Quantitative Measurement. ALP activity was assessed by a modified Great Escape SEAP Chemiluminescence Assay (BD Clontech, Mountain View, CA, USA) and/or histochemical staining assay (using a mixture of $0.1 \mathrm{mg} / \mathrm{mL}$ of naphthol AS-MX phosphate and $0.6 \mathrm{mg} / \mathrm{mL}$ of fast blue BB salt) $[13,14]$. Cultured MDSCs were seeded in 24-well plate with subconfluent of $30 \%$ and infected with AdBPM9 (experimental group), AdBPM2 (positive control group), and Ad-GFP (negative control group), respectively. At 5, 7, and 9 days after infection, ALP activity would be measured and histochemical staining would be done as indicated. The results were repeated in at least three independent experiments. ALP activity was normalized by total cellular protein concentrations among the samples.

2.2.4. Calcium Salt Sedimentation Experiment. MDSCs were seeded into a 24-well plate and infected with AdBPM9,
AdBMP2, and Ad-GFP. Alizarin red S staining was subjected at 14 days after infection. Cells were fixed with $0.05 \%(\mathrm{v} / \mathrm{v})$ glutaraldehyde at room temperature for 10 minutes. After being washed with distilled water, fixed cells were incubated with $0.4 \%$ alizarin red S (Sigma-Aldrich) for 5 minutes, followed by extensive washing with distilled water. The staining of calcium mineral deposits was recorded under bright-field microscopy.

\subsubsection{Preparation of Bone Defect Model and Animal Grouping.} Anesthetize 36 New Zealand white rabbits, then dissection was cut (longitudinally approximately $30 \mathrm{~mm}$ along the inner side of the forearm) to adequately expose the middle segment of the radius, and prepare a bone defect model of approximately $12 \mathrm{~mm}$ by an electric saw, in accordance with the length of the implant. Randomly assign the rabbits into 3 groups and implant Adv-hBMP9 + nanohydroxyapatite/polyamide bone cement, Adv-hBMP2 + nanohydroxyapatite/polyamide bone cement, and Adv-GFP + hydroxyapatite/polyamide bone cement at the left and right bone defect sites, respectively. Suture the wound layer by layer and wrap with sterile dressings. After the surgery, each rabbit in each group is injected with gentamicin sulfate $10,000 \mathrm{U}$, for $3 \mathrm{~d}$; the rabbits of each group are fed in different cages.

2.2.6. Detection by X-Ray Radiography. Carry out X-ray radiography in weeks $2,4,8,12$, and 16 after the surgery, respectively.

2.2.7. Collection and Detection of Histological Specimens. In Weeks 2, 4, 6, 8, 12, and 16 after the surgery, sacrifice 2 rabbits of each group, respectively, macroscopically observe the healing condition of the implant and surrounding tissues, and carry out a histological examination of the tissue in the stent.

\section{Results}

3.1. Separation and Cultivation of MDSCs. The cells of early wall-adhering growth (PP1) are mostly fibroblasts, with large body and with rapid growth, without significant directional growth. The cells of late-stage wall-adhering growth (PP6) are mostly short, fusiform, polygonal cells (Figure 1(a)), with obviously directional growth that may form into a long chain; however, their growth is slower than that of fibroblasts. In 7-10 d later, intercellular lamellar fusion occurs; hereafter, cell growth rate is slower than before.

3.2. Identification of MDSCs. Sca-1 is one of the most characteristic phenotypes of MDSCs; when the in vitro culture density of cells reached $70 \%-80 \%$, Sca- 1 immunohistochemical staining was carried. The results showed that $80 \%-90 \%$ of cells were positive in Sca-1 staining (Figure 1(b)).

3.3. ALP Staining and ALP Activity of BMP9 Induced in Muscle-Derived Stem Cells. ALP is the early marker for osteogenous differentiation of cells. ALP staining of MDSCs transfected with BMP9, BMP2, and GFP at day 9 was shown (Figure 2(a)). It suggested that ALP expression induced by 


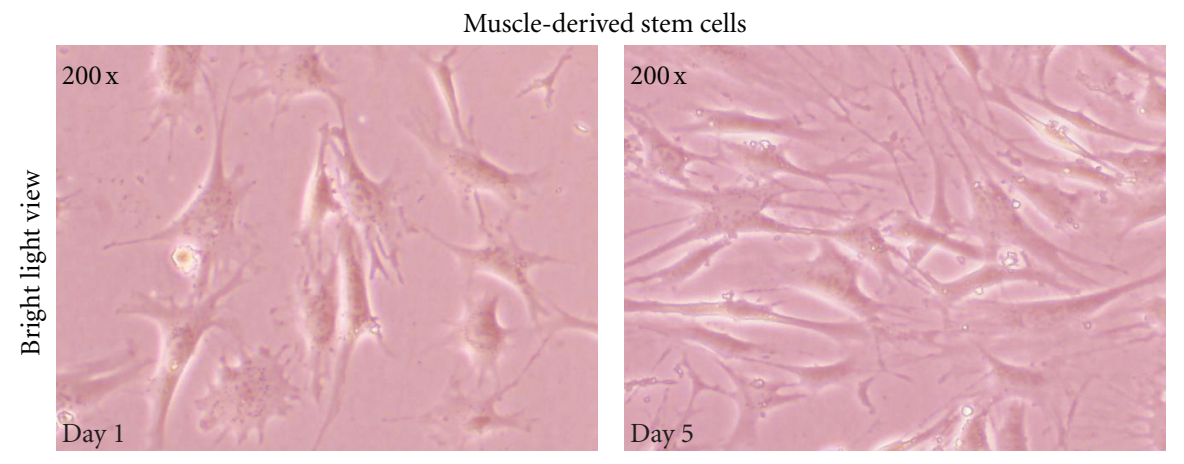

(a)
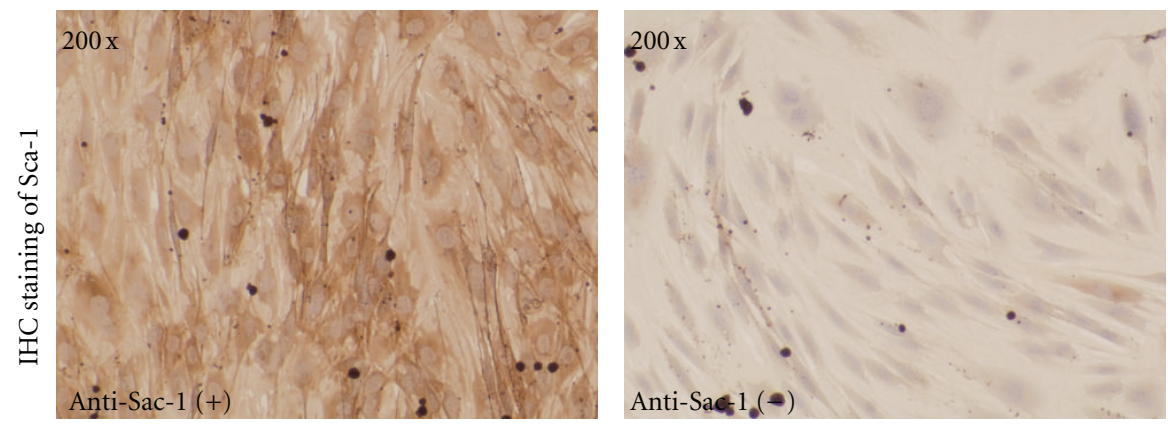

(b)

FIGURE 1: (a) Culture of muscle-derived stem cells. MDSCs were cultured and subcultured at confluent of $80 \%$. Bright view images were taken at day 1 and day 5 after subconfluent. (b) Immunohistochemical staining of Sca-1. MDSCs were subcultured. Expression of Sca-1 was assessed by immunohistochemical staining analysis at day 2 using an anti-Sca-1 antibody (Wuhan Boster Biological Technology Co., Ltd.).

BMP9 in MDSCs was higher than other groups. And also, ALP activities of MDSCs after infected by BMP9, BMP2, and GFP expression adenovirus were assessed at days 5, 7, and 9 (Figure 2(b)). ALP activities induced by BMP9 in MDSCs were significantly higher in indicated time points.

\subsection{Expression of BMP9-Induced Calcium Salt Sedimentation} in Muscle-Derived Stem Cells. Alizarin red S staining was carried out at day 14 after infection; it was found that, under the induction of BMP9, obvious calcium salt sedimentation occurred in MDSCs, the density of calcification was higher than other groups, and at the same time, the size of calcification in BMP9 group was larger (Figure 3).

3.5. Results of X-Ray Radiography. Week 2 after the surgery: no obvious callus formation was seen around the implant in each group.

Week 4 after the surgery: the boundary between the broken ends of bone and the implant was veiled in the BMP9 group, and the transmittancy of the implant was decreased. No obvious changes occurred in the remaining two groups.

Week 8 after the surgery: callus grew into the implant from the two broken ends of bone and from the side of the ulna (Figure 4). The amount of formed callus in the BMP2 group was less than that in the phase in the BMP9 group (Figure 4). A small amount of callus was seen in the side of ulna in the GFP group (Figure 4).

Week 12 after the surgery: in the BMP9 group, the two broken ends of bone were completely connected, and the majority of bone marrow cavities were recanalized. However, the two broken ends of bone were not completely connected in the BMP2 group.

Week 16 after the surgery: in the BMP9 group, the two broken ends of bone were completely connected, and the majority of bone marrow cavities were recanalized (Figure 4). However, the ends of bone broken were not completely connected in the BMP2 group (Figure 4). In the GFP group, there was only a certain amount of formed callus in the side of ulna and in the proximal end of bone broken (Figure 4).

3.6. Morphologic Macroscopic Observation. Week 2 after the surgery: in the BMP9 group, the implant was wrapped by noncohesive fibrous tissues, the two ends of the implant were connected with the recipient bone fibers, a small amount of soft tissues with blood vessels grew into the lateral aperture of the implant, no obvious new bone formation was seen, and the adhesion between the implant and the recipient bone was not firm and the implant could be shaken. In the BMP2 group, it was seen that the implant was wrapped by noncohesive fibrous tissues, no obvious new bone formation was seen, and the implant could be shaken. In the GFP group, it was seen that the implant was wrapped by a small amount of fibrous tissues.

Week 4 after the surgery: in the BMP9 group, the implant was wrapped by fibrous tissues, the proximal and distal ends of the implant were connected with the recipient bone and could not be shaken, a large amount of soft tissues with blood 
Alkaline phosphatase (histochemical) staining

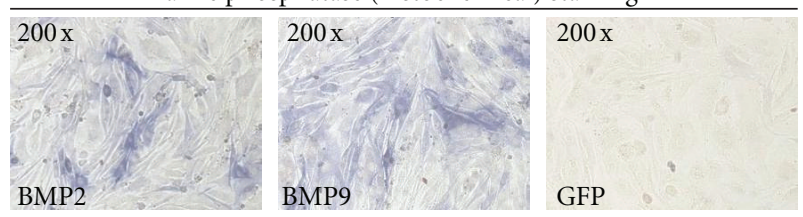

(a)

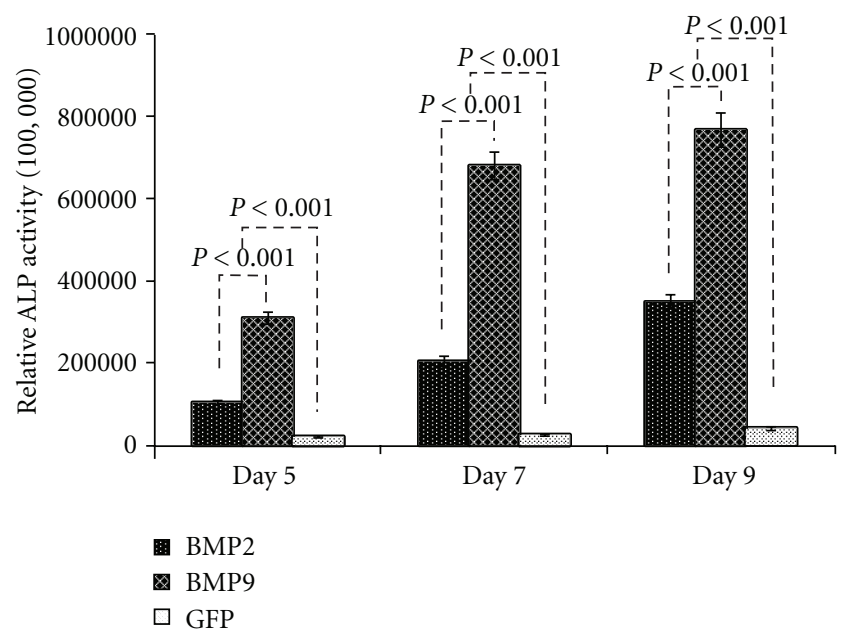

(b)

Figure 2: Potentiation of BMP-induced early osteogenetic marker ALP activity in muscle-derived stem cells. (a) ALP staining in MDSCs. MDSCs were infected with AdBMP9, AdBMP2, and AdGFP. ALP staining was done at the indicated time points. ALP expression induced by BMP9 in MDSCs was shown higher than other groups. (b) BMP-induced ALP activity in MDSCs. MDSCs were infected with AdBMP9, AdBMP2, and Ad-GFP. ALP activity was measured at the indicated time points. Each assay condition was dose in triplicate. ALP activities induced by BMP9 in MDSCs were significantly higher in indicated time points $(P<0.001)$.

vessels grew into the lateral aperture of the implant, and there was a small amount of formed new callus. In the BMP2 group, a small amount of soft tissues with blood vessels grew into the lateral aperture of the implant, and no new bone formation was seen. In the GFP group, a small amount of soft tissues with blood vessels grew into the lateral aperture of the implant, and no new bone formation was seen.

Week 6 after the surgery: in the BMP9 group, the implant was wrapped completely by fibrous membranous tissues, and there were hard new bone and callus formation. In the BMP2 group, there was a small amount of formed new callus. In the GFP group, new bone callus formation occurs initially.

Week 8 after the surgery: in the BMP9 group, there was a large amount of formed new callus. It was seen that hard calluses grew into the implant, and a bone-wedge-like structure was formed in the lateral aperture of the implant. In the BMP2 group, the lumen was full of connective tissues, and there was new callus formation. In the GFP group, a small amount of new callus was formed.

Weeks 12 and 16 after the surgery: in the BMP9 group, callus was increased. In the BMP2 group, it was seen that hard calluses grew into the implant. In the GFP group, a large amount of new callus was formed.

3.7. Results of Histological Examination. In the BMP9 group: in Week 2 after the surgery, it was seen that connective tissues grew into the lumen of the implant, and there were much inflammatory cell infiltration and immature capillary formation. In week 4 after the surgery, the lumen was full of connective tissues, inflammatory cells were reduced, a mature vascular net was formed, and it was seen that a small amount of cartilage grew. In week 6 after the surgery, a mature vascular net was formed, and there was fibrous callus formation in the stent. In week 8 after the surgery, the lumen was full of mature vascular net and cartilages, and there were much fibrous callus and osteoid formations, and a bone wedge-like structure was formed in the lateral aperture of the implant. Some cartilages were directly adhered to the surface of the implant (Figure 5). In week 12 after the surgery, the lumen was full of cartilages; after bone formation and moulding, mature bone trabecular formation was seen, similar to peripheral bones, observed under microscope, and was connected with the recipient bone mutually. In week 16 after the surgery, the bone cavity was filled with mature trabecular bone, and immature trabecular bone between meshes were connected into mesh-like and plate-like shapes (Figure 5).

In the BPM2 group: vasoformation and bone formation were similar to those in the BMP9 group; however, the times for vasoformation and bone formation were approximately 2 weeks later than those in the BMP9 group (Figure 5).

In the GFP group: the times for vasoformation and bone formation were much later than those in the BMP2 group (Figure 5).

\section{Discussion}

There are several classes of cell populations in the skeletal muscle, of which the muscle-derived stem cells (MDSCs) have multidirectional differentiation ability [4, 15-17]. In the experiment, MDSCs were extracted by the preplate technique; the principle of this technique was based on different times for wall-adhering growth of different cells: the majority of cells of initial wall-adhering growth are fibroblasts (PP1), the main portion of cells of wall-adhering growth in $24-48 \mathrm{~h}$ are satellite cells (PP2-PP4), and cells of last wall-adhering growth were Sca-1 staining-positive MDSCs. Prior to wall-adhering growth, MDSCs were of small and round spherical shape, with strong refractivity, and were also of round shape at the time of initial wall-adhering growth; after spontaneous growth, they are fused into mature polynuclear myotubes. It is different from fibroblasts in this point. After MDSCs transfected with BMP9 and BMP2, it is found by ALP staining and quantitative analysis that BMP9 induced ALP activities of muscle-derived stem cells, and the activities were increased with time, reaching peak in day 9, suggesting that MDSCs had the osteogenous differentiation ability in vitro. Meanwhile, the ALP activities induced by BMP9 were obviously higher than those in the BMP2 group, suggesting that BMP9 had higher potential ability of osteogenesis stimulating than BMP2. The results 

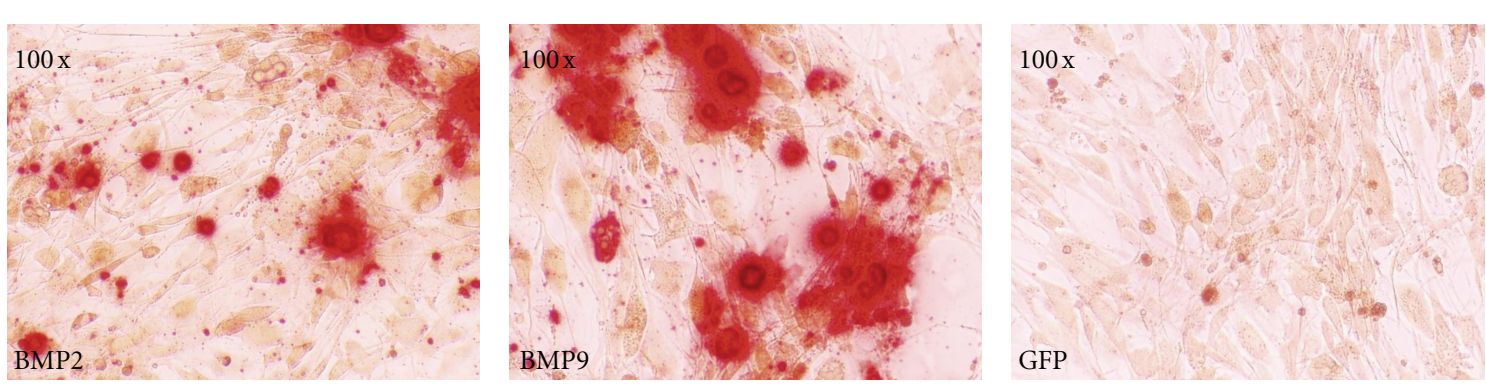

FIGURE 3: Alizarin red S staining. MDSCs were infected with adenoviruses as indicated. Alizarin red S staining was conducted at 14 days. The density of calcification induced by BMP9 was higher than other groups, and at the same time, the size of calcification in BMP9 group was larger.
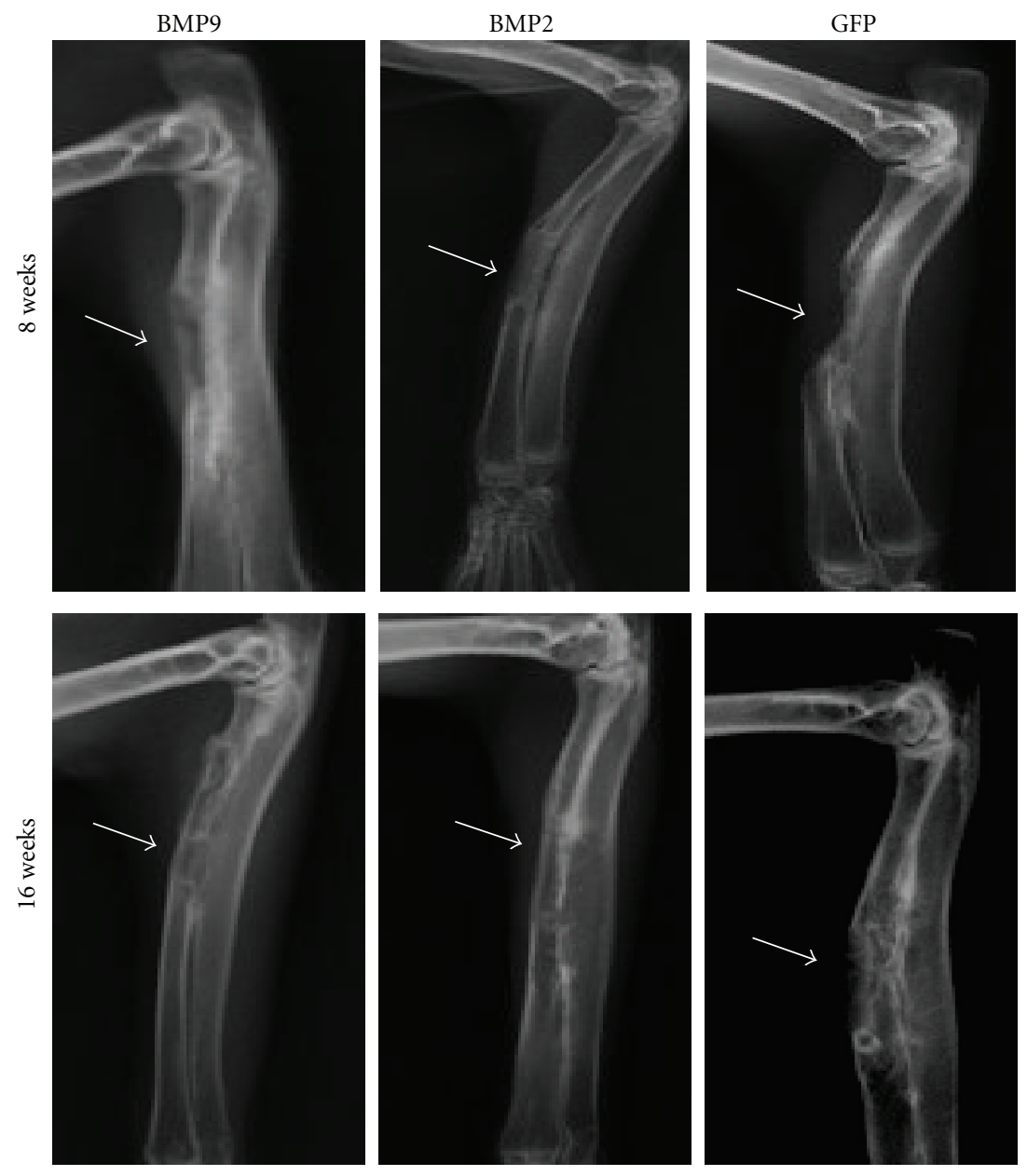

FIGURE 4: Bone formation of MDSCs induced by BMP2 and BMP9 in rabbit radius defects repairing test. BMP9 or BMP2 expression MDSCs were implanted in rabbit radius defects model. X-ray images of bone formation by MDSCs implantation assay had been taken at week 8 and week 16. Representative X-ray images are shown as indicated.

of the later-stage marker, that is, calcium salt sedimentation, for osteogenous differentiation of cells showed that there was obvious calcium salt sedimentation in the BMP9 group; moreover, compared with that in the BMP2 group, BMP9 has stronger ability of stimulating calcium salt sedimentation in MDSCs. These demonstrated that BMP9 was probably a more efficient osteoinductive growth factor compared with BMP2. The result was consistent with that from the studies conducted by Wada et al. [18].

Previously, the studies by D. S. Musgrave found that, under the action of BMP2, MDSCs resulted in ectopic osteogenesis in upper limbs [19]. In the studies by V. Wright, 

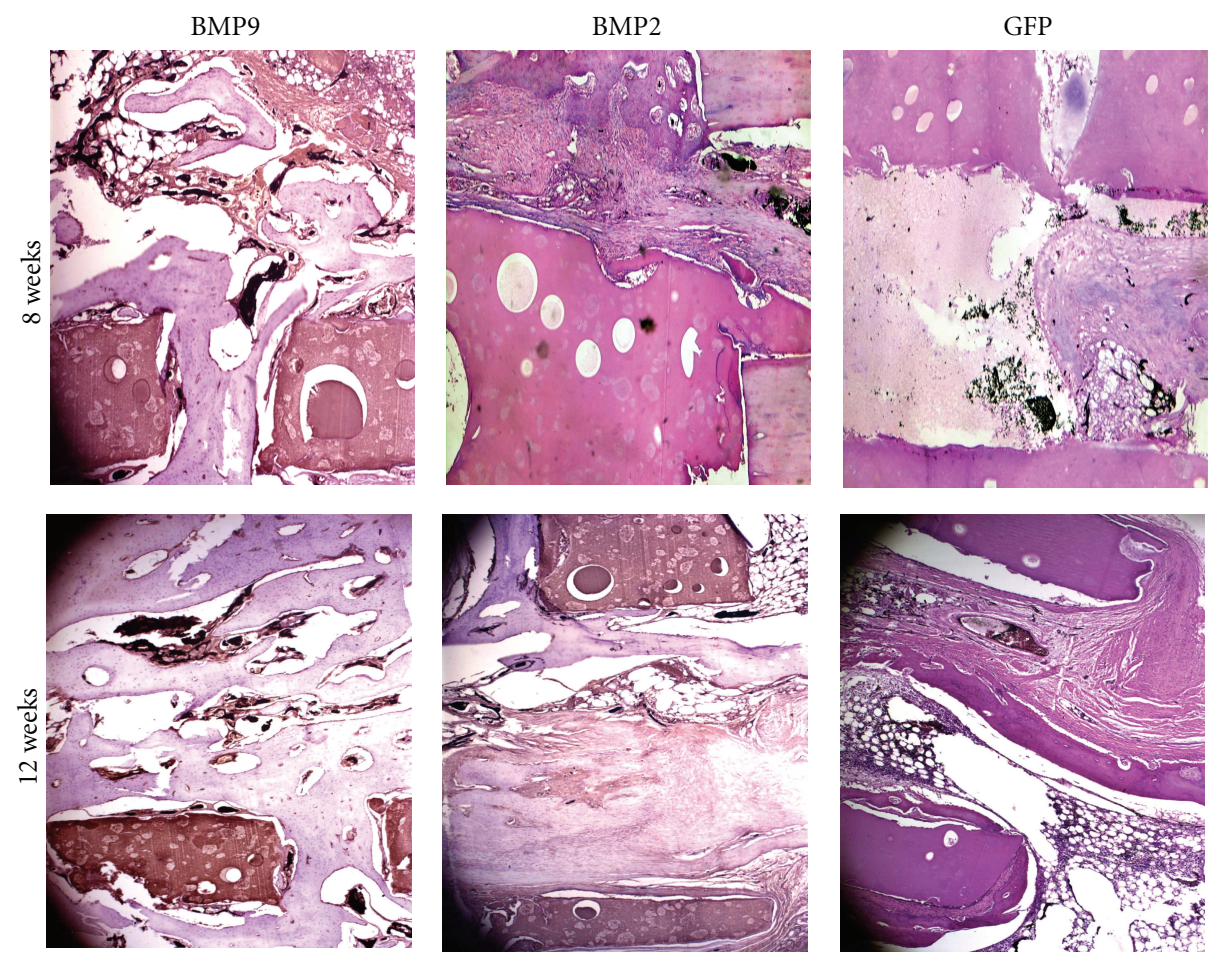

FIGURE 5: Histologic evaluation of the retrieved bone samples. Retrieved bone sample from BMP9- or BMP2-treated groups were fixed and decalcified. The paraffin-embedded sections was subjected to hematoxylin and eosine (H\&E) staining. Representative images are shown. Magnification, 200.

MDSCs transfected with BMP4-induced ectopic osteogenesis and promoted bone healing [20]. Therefore, during our study, the effects of BMP9/2/GFP in repairing radius defect were observed by using the method in which, after the middle segment defect model of radius was prepared, Adv-hBMP9/2/GFP + nanohydroxyapatite/polyamide bone cement was implanted. As a result, new bone was initially emerged in week 4 in the BMP9 group; in the BMP9 group, by week 8 , large mounts of blood vessels, soft tissues, and bone calluses were formed, and hard calluses grew into the implant. In week 16 after the surgery, the two broken ends of bone were completely connected, and the majority of bone marrow cavities were recanalized; by histological examination, mature bone trabecular was seen, and new bone trabecular between meshes was connected into meshlike and plate-like shapes. The repairing duration of bone defects in the BMP2 group was approximately 2 weeks longer than that in the BMP9 Group. Bone defects repairing in GFP group is much more slow than that in the BMP2 group. This result is consistent with the prior reports submitted by Cheng et al. [13] and Kang et al. [14]. They demonstrated that BMP9 (in addition to BMP-2, BMP4, or BMP-7) is identified as the most potent BMPs to induce orthotopic bone formation in vivo [14].

From what have been discussed above, BMP9 has strong effects of inducing directional osteogenous differentiation of muscle-derived stem cells; compared with other stem cells in tissue engineering, the in vitro cultivation of MDSCs has the advantages of good material availability, as well as strong proliferation and differentiation abilities. Meanwhile, it has also demonstrated that it is a research direction of vast potential to carry out research on artificial bone in tissue engineering, by using highly efficient osteogenous induction factor and readily available, adequate tissue stem cells in combination.

\section{Acknowledgments}

The authors thank Tong-chuan, HE, University of Chicago Medical Center for providing the BMP9 expression adenovirus. This work was supported in part by the Natural Science Foundation of China (no. 31000434 C. Liang and no. 30772211 to D. Zhong-Liang), and the Natural Science Foundation Project of Chongqing Science and Technology Commission CSTC no. 2008BB5078 (D. Zhong-Liang) and CSTC2011jjzt0063 (C. Liang). L. Xiang and C. Liang are the co-first authors of this paper.

\section{References}

[1] C. A. Collins and T. A. Partridge, "Self-renewal of the adult skeletal muscle satellite cell," Cell Cycle, vol. 4, no. 10, pp. 1338-1341, 2005.

[2] J. T. Williams, S. S. Southerland, J. Souza, A. F. Calcutt, and R. G. Cartledge, "Cells isolated from adult human skeletal muscle capable of differentiating into multiple mesodermal phenotypes," American Surgeon, vol. 65, no. 1, pp. 22-26, 1999.

[3] R. I. Sherwood, J. L. Christensen, I. M. Conboy et al., "Isolation of adult mouse myogenic progenitors: functional heterogeneity of cells within and engrafting skeletal muscle," Cell, vol. 119, no. 4, pp. 543-554, 2004. 
[4] J. Y. Lee, Z. Qu-Petersen, B. Cao et al., "Clonal isolation of muscle-derived cells capable of enhancing muscle regeneration and bone healing," Journal of Cell Biology, vol. 150, no. 5, pp. 1085-1099, 2000.

[5] R. Kuroda, A. Usas, S. Kubo et al., "Cartilage repair using bone morphogenetic protein 4 and muscle-derived stem cells," Arthritis and Rheumatism, vol. 54, no. 2, pp. 433-442, 2006.

[6] S. Govender, C. Csimma, H. K. Genant et al., "Recombinant human bone morphogenetic protein-2 for treatment of open tibial fractures a prospective, controlled, randomized study of four hundred and fifty patients," Journal of Bone and Joint Surgery A, vol. 84, no. 12, pp. 2123-2134, 2002.

[7] Y. Acil, I. N. Springer, and V. Broek, "Effects of bone morphogenetic protein-7 stimulation on osteoblasts cultured on different biomaterials," Journal of Cellular Biochemistry, vol. 86, no. 1, pp. 90-98, 2002.

[8] H. S. Sandhu, "Bone morphogenetic proteins and spinal surgery," Spine, vol. 28, no. 15, supplement, pp. S64-S73, 2003.

[9] I. Muñoz-Sanjuán and A. H. Brivanlou, "Neural induction, the default model and embryonic stem cells," Nature Reviews Neuroscience, vol. 3, no. 4, pp. 271-280, 2002.

[10] X. Cao and D. Chen, "The BMP signaling and in vivo bone formation," Gene, vol. 357, no. 1, pp. 1-8, 2005.

[11] D. Laurent, M. Christine, M. Sabine, J. J. Feige, and S. Bailly, "Identification of BMP9 and BMP10 as functional activators of the orphan activin receptor-like kinase 1 (ALK1) in endothelial cells," Blood, vol. 109, no. 5, pp. 1953-1961, 2007.

[12] Z. Qu-Petersen, B. Deasy, R. Jankowski et al., "Identification of a novel population of muscle stem cells in mice: potential for muscle regeneration," Journal of Cell Biology, vol. 157, no. 5, pp. 851-864, 2002.

[13] H. Cheng, W. Jiang, F. M. Phillips et al., "Osteogenic activity of the fourteen types of human bone morphogenetic proteins (BMPs)," Journal of Bone and Joint Surgery A, vol. 85, no. 8, pp. 1544-1552, 2003.

[14] Q. Kang, M. H. Sun, H. Cheng et al., "Characterization of the distinct orthotopic bone-forming activity of 14 BMPs using recombinant adenovirus-mediated gene delivery," Gene Therapy, vol. 11, no. 17, pp. 1312-1320, 2004.

[15] B. Peault, M. Rudnicki, Y. Torrente et al., "Stem and progenitor cells in skeletal muscle development, maintenance, and therapy," Molecular Therapy, vol. 15, no. 5, pp. 867-877, 2007.

[16] S. L. McKinney-Freeman, K. A. Jackson, F. D. Camargo, G. Ferrari, F. Mavilio, and M. A. Goodell, "Muscle-derived hematopoietic stem cells are hematopoietic in origin," Proceedings of the National Academy of Sciences of the United States of America, vol. 99, no. 3, pp. 1341-1346, 2002.

[17] A. Asakura, M. Komaki, and M. Rudnicki, "Muscle satellite cells are multipotential stem cells that exhibit myogenic, osteogenic, and adipogenic differentiation," Differentiation, vol. 68, no. 4-5, pp. 245-253, 2001.

[18] M. R. Wada, M. Inagawa-Ogashiwa, S. Shimizu, S. Yasumoto, and N. Hashimoto, "Generation of different fates from multipotent muscle stem cells," Development, vol. 129, no. 12, pp. 2987-2995, 2002.

[19] D. S. Musgrave, P. Bosch, J. Y. Lee et al., "Ex vivo gene therapy to produce bone using different cell types," Clinical Orthopaedics and Related Research, no. 378, pp. 290-305, 2000.

[20] V. Wright, H. Peng, A. Usas et al., "BMP4-expressing musclederived stem cells differentiate into osteogenic lineage and improve bone healing in immunocompetent mice," Molecular Therapy, vol. 6, no. 2, pp. 169-178, 2002. 

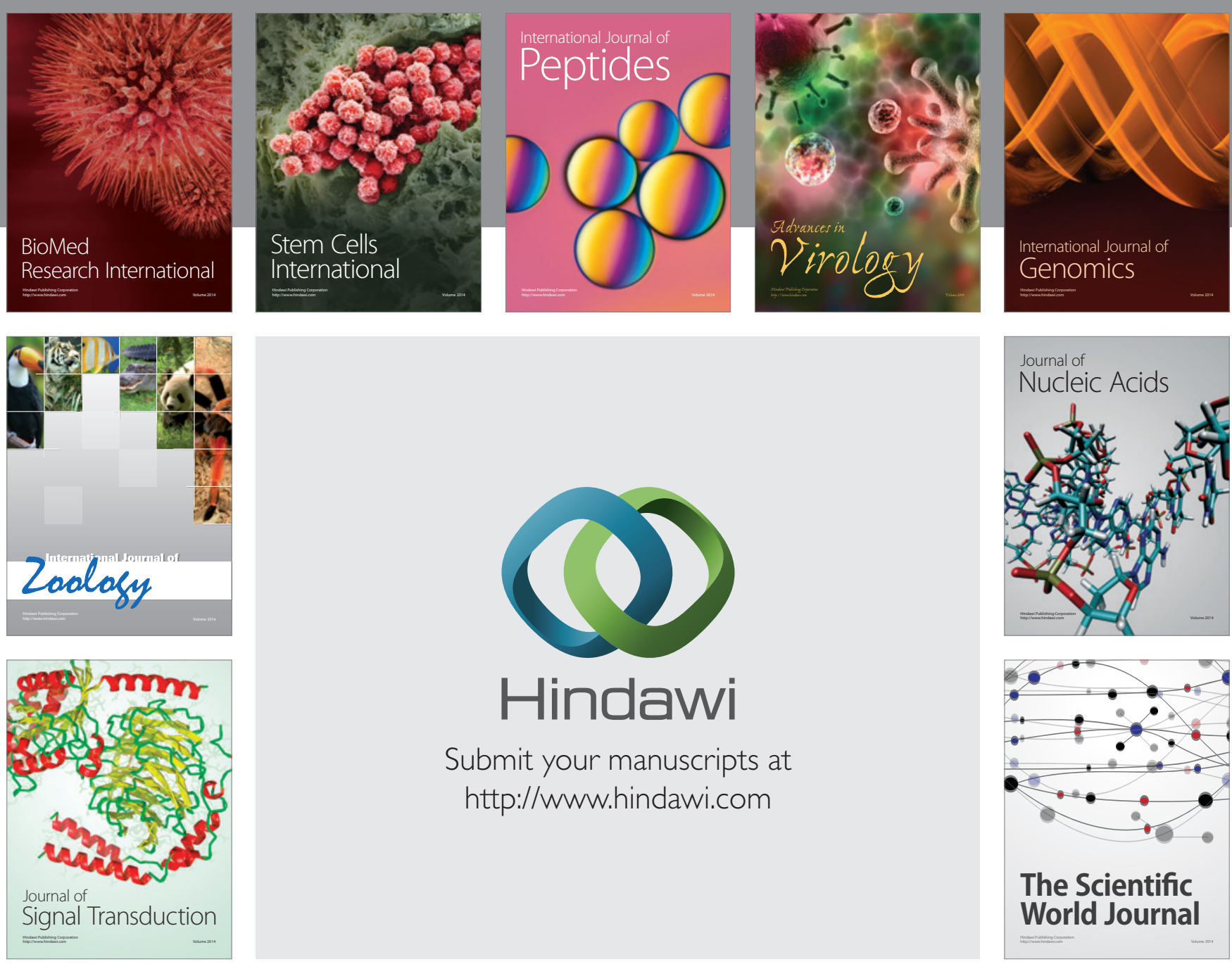

Submit your manuscripts at

http://www.hindawi.com
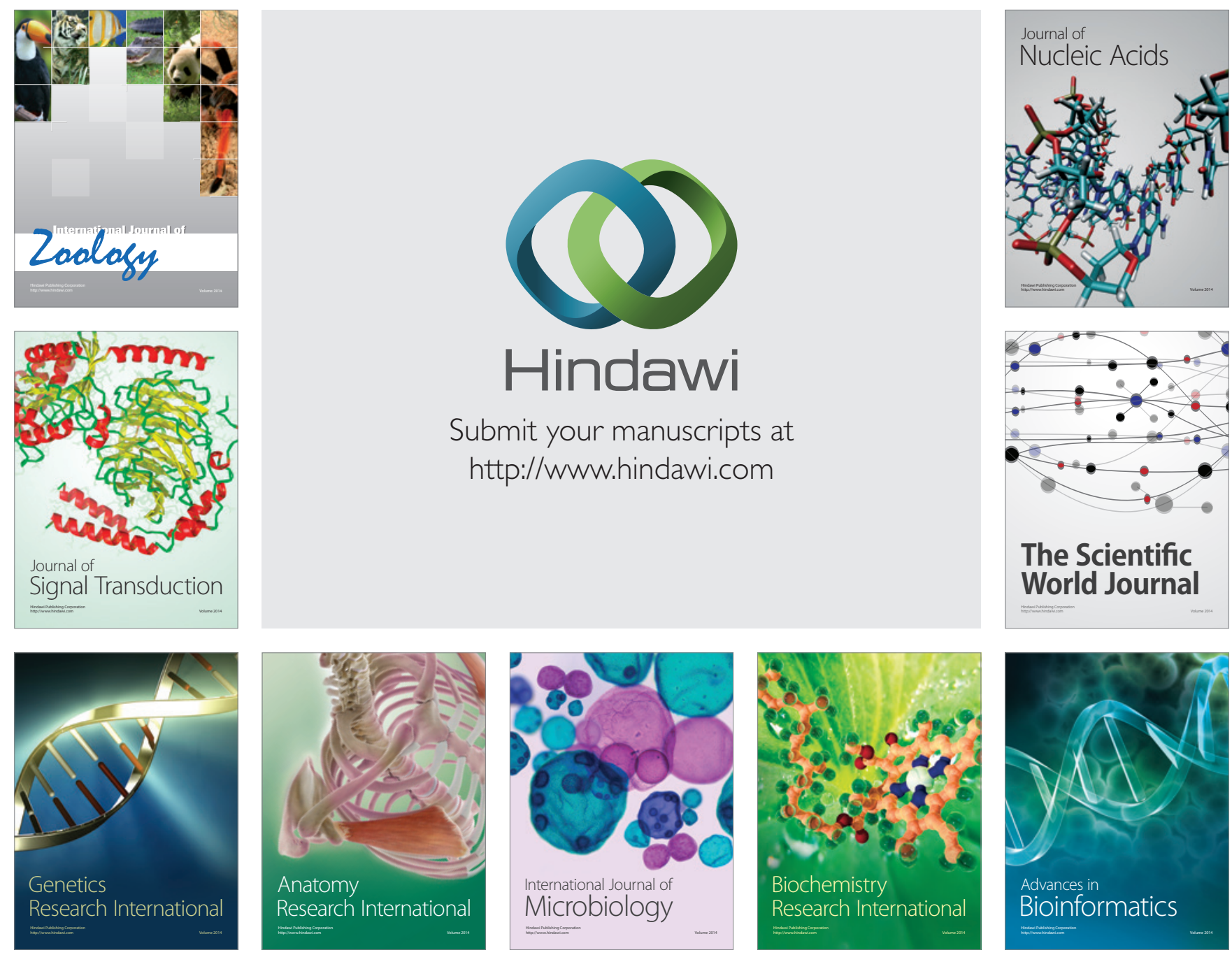

The Scientific World Journal
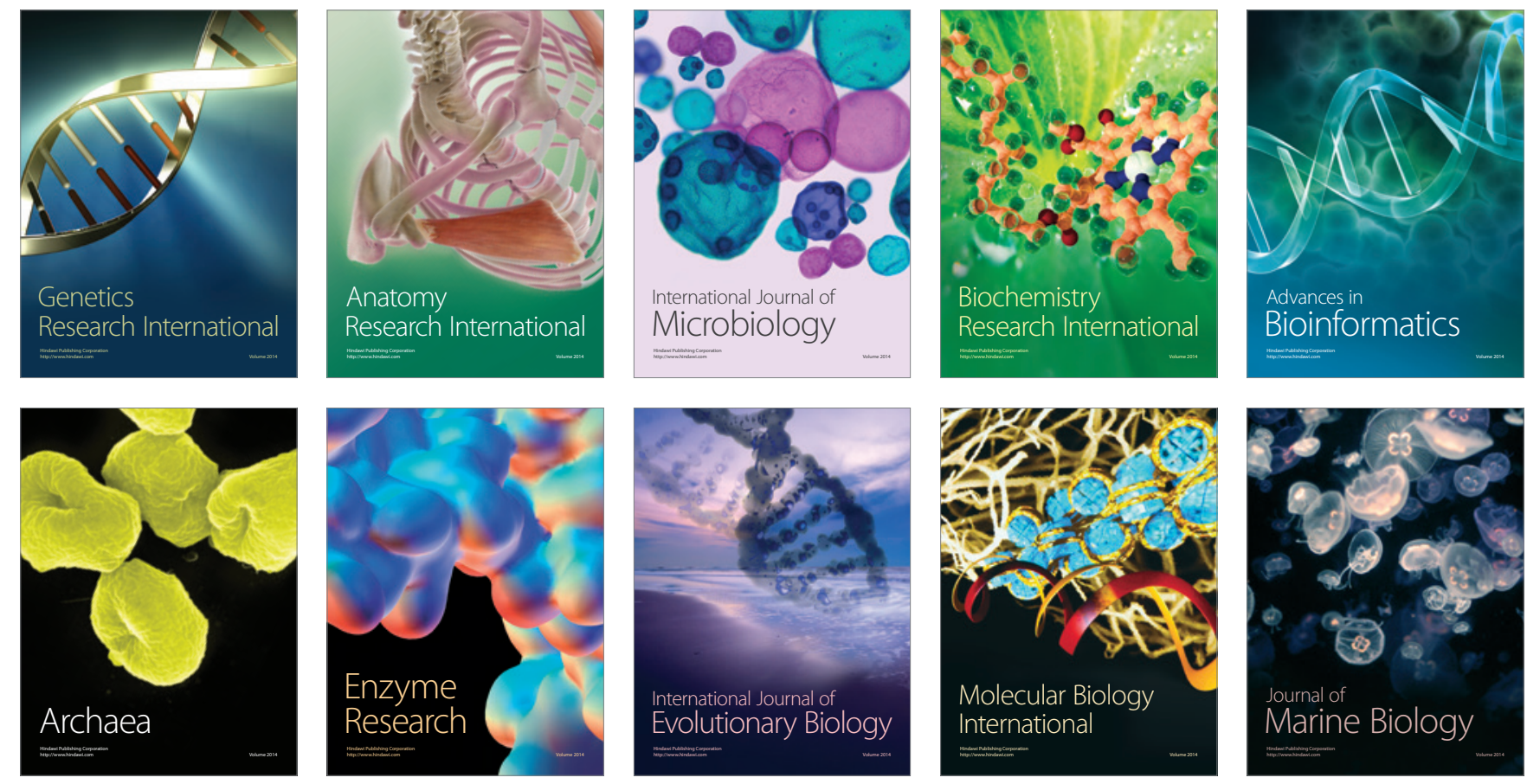